\title{
The Bacillus subtilis dnaC gene encodes a protein homologous to the DnaB helicase of Escherichia coli
}

\author{
Yumi Sakamoto, ${ }^{1}$ Sumiko Nakai, ${ }^{2}$ Shigeki Moriya, ${ }^{1}$ Hiroshi Yoshikawa ${ }^{3}$ \\ and Naotake Ogasawara'
}

Author for correspondence: Naotake Ogasawara. Tel: +8174372 5430. Fax: +81743725439.
e-mail: nogasawa@bs.aist-nara.ac.jp

1.3 Departments of Cell Biology ${ }^{1}$ and Molecular Biology ${ }^{3}$, The Graduate School of Biological Sciences, Nara Institute of Science and Technology, 8916-5, Takayama, Ikoma, Nara 630-01, Japan

2 Department of Genetics, Osaka University Medical School, 2-2 Yamadaoka, Suita, Osaka 565, Japan

\begin{abstract}
Within the region of the Bacillus subtilis chromosome assigned to us in the genome sequencing project, we found a gene, the product of which is similar to the DnaB protein (replicative DNA helicase) of Escherichia coli. Three B. subtilis dna gene mutations, dnaC30 and ts56 causing defects in elongation and ts199 causing a defect in the initiation of replication, were mapped in the gene by transformation and DNA sequencing. Both dnaC30 and ts56 have been located near the amino-terminal end of the B. subtilis DnaC protein. In contrast, ts 199 has been located near the carboxy-terminal of the protein. Our results indicate that the $B$. subtilis dnaC gene encodes a counterpart of the $E$. coli dnaB helicase.
\end{abstract}

Keywords: Bacillus subtilis, genome sequencing, $d_{n} C$, DNA helicase
The DnaB protein of Escherichia coli acts as a central replicative DNA helicase and therefore is essential for initiation and elongation in chromosome replication. In Bacillus subtilis, its counterpart has not yet been identified. We have recently found a gene (ORF454) near the pur $A$ gene in the $180 \mathrm{~kb}$ region assigned to us as part of the $B$. subtilis genome sequencing project, the deduced amino acid sequence of which shows $42 \%$ identity to $E$. coli DnaB helicase (Ogasawara et al., 1994). The organization of ORFs around this gene is shown in Fig. 1. The dnaC mutation and two other groups of dna-ts mutations ( $t s A$ and $t s B$ ) have already been mapped near pur $A$ on the $B$. subtilis genetic map (Karamata \& Gross, 1970; Bazill \& Retief, 1969; Andersen \& Ganesan, 1975; Hara \& Yoshikawa, 1973). The $d n a C$ and $t s A$ mutants were defective in elongation, whereas $t s B$ mutants were defective in initiation. Although $d n a C$ and ts $A, B$ were mapped at different but close loci on the $B$. subtilis genetic map (Anagnostopoulos et al., 1993), the relationship between $d n a C$ and $t s A, B$ was not examined (Yoshikawa \& Wake, 1993). To find which of the dna mutations were located on ORF454, three dna mutant strains [ts56 and ts 199 (representatives for the ts $A$ and $t s B$ group, respectively) and dnaC30] were used as recipients for transformation. Donor DNA was derived from four $\lambda$ clones (D3, D25, PL and PL2-3). As shown in Fig. 1, the

The DDBJ accession number for the nucleotide sequence reported in this paper is D26185.
DNA of three clones (D3, D25 and PL) did not revert the temperature-sensitive phenotype of any mutant, whereas PL2-3 DNA transformed all mutants with significantly high efficiency. These results indicate that the three markers are located close to each other and in the vicinity of ORF454. To map these mutation sites more precisely, the DNA of three other clones (PL2, YS1 and YS2) was assayed for its ability to complement them. Fig. 1 shows that the DNA of all three clones transformed $d n a C 30$ and ts56 mutant cells, indicating that both mutations are located on a common fragment (from an EcoRI site in ORF454 to a HindIII site in ORF66). In contrast, when a ts199 strain was used as recipient, only YS1 DNA had transforming activity, suggesting that the $t s 199$ mutation is located in the $3^{\prime}$ region of ORF454. These results suggest that all three dna mutations are located in ORF454.

The $B$. subtilis pur $A$ gene was cloned based upon its sequence similarity to the $E$. coli counterpart (Mantsala \& Zalkin, 1992). In this study we have confirmed that the $B$. subtilis pur $A 16$ mutation is situated in a sequence containing this gene (Fig. 1).

As both $d n a C 30$ and $t 556$ were shown to be present in an EcoRI-HindIII DNA fragment of $880 \mathrm{bp}$, the nucleotide sequence of the region in both mutant chromosomes was determined to identify these mutation sites. Furthermore, the nucleotide sequence of the $2.15 \mathrm{~kb}$ fragment (corresponding to the insert in clone YS1) of the $t 5199$ chromosome was determined to identify the $t 5199$ 


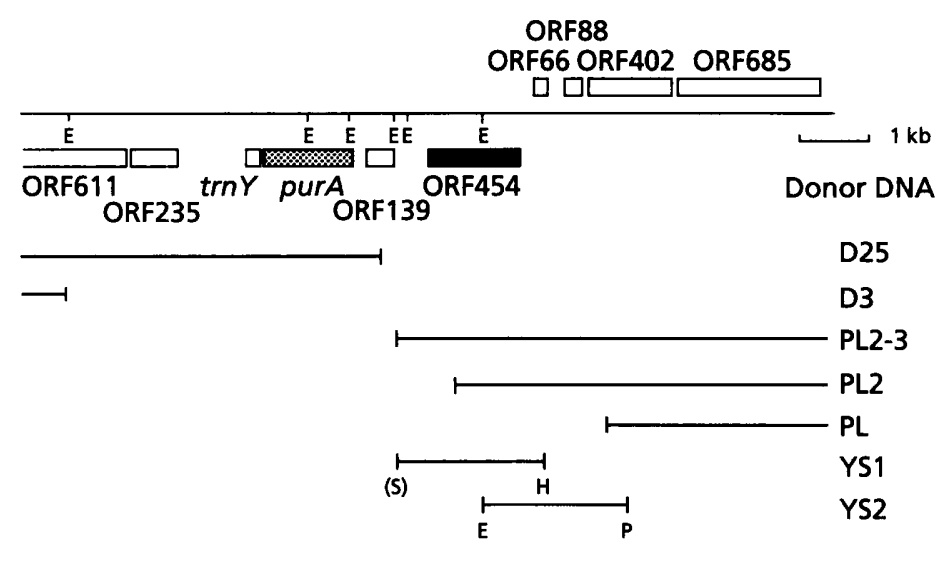

\begin{tabular}{|c|c|c|c|}
\hline \multicolumn{4}{|c|}{ No. of transformants } \\
\hline \multicolumn{3}{|c|}{$\mathbf{A}$} & B \\
\hline dnac30 & ts56 & $s 199$ & $\begin{array}{l}\text { CRK6000 } \\
\text { (purA16) }\end{array}$ \\
\hline 0 & 0 & 0 & $1 \times 10^{4}$ \\
\hline 0 & 0 & 0 & 0 \\
\hline 3000 & 664 & 342 & - \\
\hline - & 555 & 0 & - \\
\hline 0 & 0 & 0 & - \\
\hline 500 & 125 & 361 & - \\
\hline 500 & 163 & 0 & - \\
\hline
\end{tabular}

\begin{abstract}
Fig. 1. Location of the dnaC30, ts56 and ts 199 mutations on the $B$. subtilis chromosome determined by transformation with cloned DNA fragments. Gene organization and a restriction map of a region containing a gene (ORF454) homologous to $E$. coli dnaB are schematically illustrated at the top left half of the figure. Boxes represent ORFs. Genes transcribed from left to right are shown above the map, and those transcribed in the opposite direction below the map. In the lower part, five $\lambda$ clones (PL, PL2, PL2-3, D3 and D25) obtained from two partially Sau3A-digested chromosomal DNA libraries of $B$. subtilis (Ogasawara et al., 1994) and two M13 subclones (YS1 and 2) derived from PL2-3 are shown as chromosomal DNA inserts in phage clones used for transformation. Abbreviations for restriction sites are as follows: $E$, EcoRI; H, HindIII; P, Pvull. (S) indicates that the Sall site in the $\lambda$ vector portion of PL2-3 DNA was used for subcloning into M13. On the right half of the figure, the number of transformants present in $10 \mu \mathrm{l}$ of competent cell suspension is shown. Four mutant strains, dnaC30 (Itaya, 1993), ts56 and ts199 (Hara \& Yoshikawa, 1973), and purA16 (Moriya et al., 1990) were used as recipients to identify the loci of the mutations. Transformation of competent $B$. subtilis cells was performed as described previously (Haas \& Yoshikawa, 1969). The transforming activity of each donor DNA $\left(1 \mu \mathrm{g} \mathrm{ml} \mathrm{m}^{-1}\right)$ is shown as the mean number of temperature-resistant transformants selected at $48{ }^{\circ} \mathrm{C}$ in $\mathrm{A}$ and purA $A^{+}$transformants grown on plates lacking adenine in B.
\end{abstract}

mutation site. In each case, a single base pair change was found in comparison with the wild-type sequence and the change caused a missense mutation in the coding region of ORF454. Thus, aspartic acid (residue 42) was replaced by glycine (codon change from G $\underline{A T}$ to $\mathrm{G} \underline{\mathrm{GT}}$ ) in $t s 56$, threonine (residue 69) was replaced by isoleucine (ACA to $\mathrm{ATA}$ ) in dna C 30 and alanine (residue 350 ) was replaced by valine (GLG to GTG) in ts199. These results demonstrate that ORF454, encoding a protein similar to E. coli DnaB, is identical to the $d n a C$ gene. Furthermore, both $t 556$ and $t s 199$ are mutant alleles of $d n a C$. Therefore, hereafter, we call $t s 56$ and $t s 199, d n a C 56$ and $d n a C 199$, respectively. It was also shown that two types of mutants defective in elongation ( $d n a C 30$ and $d n a C 50$ ) and in initiation (dnaC199) can be isolated, both affected by the B. subtilis $d n a C$ gene. Similarly, two types of dna mutants were reported for the $d n a B$ gene of E. coli (Wechsler \& Gross, 1971; Zyskind \& Smith, 1977).

Nakayama et al. (1984a) determined that the E. coli DnaB protein could be divided by trypsin digestion into two relatively stable domains, a $12 \mathrm{kDa}$ amino-terminal (fragment III) and a $33 \mathrm{kDa}$ carboxy-terminal domain (fragment II). About 45 amino acids between fragments II and III serve as a hinge region. In addition, 14 amino acids of the amino-terminal end are also lost during the cleavage. In Fig. 2, the amino acid sequence of B. subtilis DnaC helicase is compared with three other helicases, $E$. coli and Salmonella typhimurium dnaB helicases and B. subtilis phage SPP1 replicative DNA helicase. In previous studies, the carboxy-terminal portions of bacterial and bacteriophage primase-associated helicases were found to be conserved (Ilyina et al., 1992; Pedre et al., 1994). The DnaC protein of $B$. subtilis also showed significant similarity in its carboxy-terminal half with the three other helicases, in that the A and B motifs involved in purine NTP-binding and hydrolysis (Walker et al., 1982) were conserved (Fig. 2). Significant similarity was also found in the remaining portion among bacterial helicases, except that the amino-terminal 14 amino acids were missing in $B$. subtilis (Fig. 2).

The dnaC199 mutation identified in this study was found to be located near the carboxy-terminal. Since the dnaC199 mutant is defective in the initiation of replication, it is unlikely that ATP-binding and/or ATPase activity are also affected in this mutant, since ATP hydrolysis was shown to be essential for the helicase action of E. coli DnaB (LeBowitz \& McMacken, 1986) and the deficiency would create mutants defective in elongation, not initiation. The carboxy-terminal domain of $B$. subtilis DnaC seems to be participating in protein-protein interaction, analogous to the situation in $E$. coli where this domain in E. coli DnaB is able to form a hexamer (Nakayama et al., 1984a), and probably interact with the $\lambda$ P protein (Chang et al., 1991). The mutant DnaC199 protein might not be able to interact with a carrier protein like the E. coli DnaC protein, although the amino-terminal domain of DnaB is essential for this interaction in E. coli (Nakayama et al., 1984a). 
$\nabla$

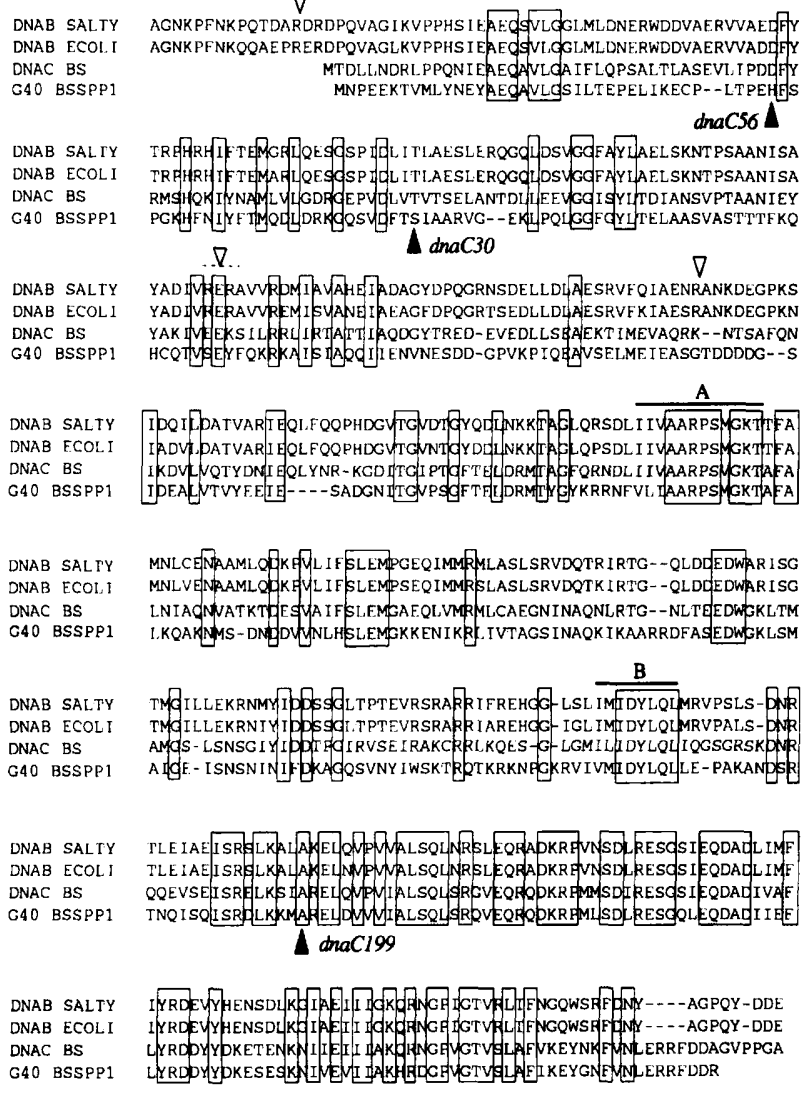

Fig. 2. Alignment of the amino acid sequences of the $B$. subtilis DnaC, E. coli DnaB (Nakayama et al., 1984b), S. typhimurium DnaB (Wong et al., 1988) and B. subtilis phage SPP1 G40 DNA helicases (Pedre et al., 1994). The alignment of the amino acid sequences was performed with the GENEWORKS program (IntelliGenetics). Identical amino acids are boxed. Identities between two of the four proteins were as follows: $93 \%$ between $E$. coli DnaB and S. typhimurium DnaB, $42 \%$ between E. coli DnaB and $B$. subtilis DnaC, $41 \%$ between $B$. subtilis DnaC and phage SPP1 G40 and $32 \%$ between $E$. coli DnaB and SPP1 G40 proteins. Thick lines labelled $A$ and $B$ indicate the putative motifs for NTP-binding (Walker et al., 1982). Open arrowheads indicate the amino and carboxyl ends of fragments II and III produced by trypsin digestion of $E$. coli DnaB (Nakayama et al., 1984a). Closed arrowheads indicate the mutation sites identified in this study on the amino acid sequence of the wildtype $B$. subtilis DnaC. The mutations were determined as follows. Various DNA fragments from the region identified as carrying the dna mutations were prepared by PCR using chromosomal DNA extracted from the mutants as template, followed by direct sequencing of the PCR products as described previously (Ogasawara et al., 1994).

In contrast to the high similarity of the carboxy-terminal domain in the four replicative DNA helicases, the aminoterminal domain showed less similarity (Fig. 2). The $d n a C 56$ and $d n a C 30$ mutations, defective in elongation, were located in this domain. The amino acids in the wildtype sequence corresponding to those changed in the mutants were conserved in the bacterial helicases. Nakayama et al. (1984a) suggested that the amino-terminal domain of $E$. coli DnaB is essential for interaction with primase and DnaC. Therefore, an interaction of B. subtilis
DnaC with primase might be impaired in both $d n a C 56$ and dnaC 30 mutants, resulting in a deficiency in elongation.

\section{ACKNOWLEDGEMENTS}

We thank Dr Mitsuyasu Itaya for providing the B. subtilis dnaC30 mutant. We also thank Drs Juan C. Alonso and William Firshein for critical reading of the manuscript and valuable discussions. This work was supported by a Grant-in-Aid for Creative Research (Human Genome Program) from the Ministry of Education, Science and Culture, Japan.

\section{REFERENCES}

Anagnostopoulos, C., Piggot, P. J. \& Hoch, J. A. (1993). The genetic map of Bacillus subtilis. In Bacillus subtilis and Other GramPositive Bacteria: Biochemistry, Physiology and Molecular Genetics, pp. 425-461. Edited by A. L. Sonenshein, J. A. Hoch \& R. Losick. Washington, DC: American Society for Microbiology.

Andersen, J. J. \& Ganesan, A. T. (1975). Temperature-sensitive deoxyribonucleic acid replication in a $d n a C$ mutant of Bacillus subtilis. J Bacteriol 121, 173-183.

Bazill, G. \& Retief, Y. (1969). Temperature-sensitive DNA synthesis in a mutant of Bacillus subtilis. J Gen Microbiol 56, 87-97.

Chang, S.-F., Ng, D., Baird, L. \& Georgopoulos, C. (1991). Analysis of an Escherichia coli dnaB temperature-sensitive insertion mutation and its cold-sensitive extragenic suppressor. $J$ Biol Chem 266, 3654-3660.

Haas, M. \& Yoshikawa, H. (1969). Defective bacteriophage PBSH in Bacillus subtilis. I. Induction, purification and physical properties of the bacteriophage and its deoxyribonucleic acid. $J$ Virol 3, 233-247.

Hara, H. \& Yoshikawa, H. (1973). Asymmetric bidirectional replication of Bacillus subtilis chromosome. Nature New Biol 244, 200-203.

llyina, T. V., Gorbalenya, A. E. \& Koonin, E. V. (1992). Organization and evolution of bacterial and bacteriophage primase-helicase systems. J Mol Evol 34, 351-357.

Itaya, M. (1993). Stability and asymmetric replication of the Bacillus subtilis 168 chromosome structure. J Bacteriol 175, 741-749.

Karamata, D. \& Gross, J. D. (1970). Isolation and genetic analysis of temperature-sensitive mutants of $B$. subtilis defective in DNA synthesis. Mol \& Gen Genet 108, 277-287.

LeBowitz, J. H. \& McMacken, R. (1986). The Escherichia coli DnaB replication protein is a DNA helicase. J Biol Chem 261, 4738-4748.

Mantsala, P. \& Zalkin, H. (1992). Cloning and sequence of Bacillus subtilis pur $A$ and gua $A$, involved in the conversion of IMP to AMP and GMP. J Bacteriol 174, 1883-1890.

Moriya, S., Kato, K., Yoshikawa, H. \& Ogasawara, N. (1990). Isolation of a dna $A$ mutant of Bacillus subtilis defective in initiation of replication: amount of DnaA protein determines cells' initiation potential. EMBO J 9, 2905-2910.

Nakayama, N., Arai, N., Kaziro, Y. \& Arai, K. (1984a). Structural and functional studies of the $d n a B$ protein using limited proteolysis. $J$ Biol Chem 259, 88-96.

Nakayama, N., Arai, N., Bond, M. W., Kaziro, Y. \& Arai, K. (1984b). Nucleotide sequence of $d n a B$ and the primary structure of the $d n a B$ protein from Escherichia coli. J Biol Chem 259, 97-101.

Ogasawara, N., Nakai, S. \& Yoshikawa, H. (1994). Systematic sequencing of the 180 kilobase region of the Bacillus subtilis chromosome containing the replication origin. DN $A$ Res 1, 1-14. 
Pedre, X., Weise, F., Chai, S., Luder, G. \& Alonso, J. C. (1994). Analysis of $c i$ and trans acting elements required for the initiation of DNA replication in the Bacillus subtilis bacteriophage SPP1. J Mol Biol 236, 1324-1340.

Walker, J. E., Saraste, M., Runswick, M. J. \& Gay, N. J. (1982). Distantly related sequences in the a- and b-subunits of ATP synthase, myosin, kinases and other ATP-requiring enzymes and a common nucleotide binding fold. EMBO J 1, 945-951.

Wechsler, J. W. \& Gross, J. (1971). Escherichia coli mutants temperature sensitive for DNA synthesis. Mol \& Gen Genet 113, 273-284.

Wong, A., Kean, L. \& Maurer, R. (1988). Sequence of the $d n a B$ gene of Salmonella typhimurium. J Bacteriol 170, 2668-2675.
Yoshikawa, H. \& Wake, R. G. (1993). Initiation and termination of chromosome replication. In Bacillus subtilis and Other Gram-Positive Bacteria: Biochemistry, Pbysiology and Molecular Genetics, pp. 507-528. Edited by A. L. Sonenshein, J. A. Hoch \& R. Losick. Washington, DC: American Society for Microbiology.

Zyskind, J. W. \& Smith, D. W. (1977). Novel Escherichia coli dnaB mutant: direct involvement of the dnaB252 gene product in the synthesis of an origin-ribonucleic acid species during initiation of a round of deoxyribonucleic acid replication. $J$ Bacteriol 129, $1476-1486$.

Received 1 September 1994; revised 4 November 1994; accepted 16 November 1994 